\title{
One-sided Post-processing for the Discontinuous Galerkin Method Using ENO Type Stencil Choosing and the Local Edge Detection Method
}

\author{
Rick Archibald, ${ }^{1}$ Anne Gelb, ${ }^{2}$ Sigal Gottlieb, ${ }^{3}$ and Jennifer Ryan ${ }^{4}$
}

Received December 22, 2003; accepted (in revised form) August 12, 2004; Published online May 27, 2006

In a previous paper by Ryan and Shu [Ryan, J. K., and Shu, C.-W. (2003). Methods Appl. Anal. 10(2), 295-307], a one-sided post-processing technique for the discontinuous Galerkin method was introduced for reconstructing solutions near computational boundaries and discontinuities in the boundaries, as well as for changes in mesh size. This technique requires prior knowledge of the discontinuity location in order to determine whether to use centered, partially one-sided, or one-sided post-processing. We now present two alternative stencil choosing schemes to automate the choice of post-processing stencil. The first is an ENO type stencil choosing procedure, which is designed to choose centered post-processing in smooth regions and one-sided or partially one-sided post-processing near a discontinuity, and the second method is based on the edge detection method designed by Archibald, Gelb, and Yoon [Archibald, R., Gelb, A., and Yoon, J. (2005). SIAM J. Numeric. Anal. 43, 259-279; Archibald, R., Gelb, A., and Yoon, J. (2006). Appl. Numeric. Math. (submitted)]. We compare these stencil choosing techniques and analyze their respective strengths and weaknesses. Finally, the automated stencil choices are applied in conjunction with the appropriate post-processing procedures and it is determine that the resulting numerical solutions are of the correct order.

KEY WORDS: Accuracy enhancement;post-processing; discontinuous Galerkin method; ENO; local edge detection.

AMS(MOS) subject classification: 65M60.

\footnotetext{
${ }^{1}$ Computer Science and Mathematics Division, Oak Ridge National Lab, Oak Ridge, TN.

${ }^{2}$ Department of Mathematics, Arizona State University, Tempe, AZ 85287, USA.

${ }^{3}$ Department of Mathematics, University of Massachusetts-Dartmouth, Dartmouth, MA 02747, USA.

${ }^{4}$ Department of Mathematics, Virginia Tech, 460 McBryde, Blacksburg, VA 24061-123. E-mail: jkryan@vt.edu.
} 


\section{INTRODUCTION}

In a previous paper by Ryan and Shu [20], a one-sided post-processing technique for the discontinuous Galerkin method was introduced for reconstructing solutions near computational boundaries and discontinuities in the solution, as well as for changes in mesh size. The technique requires a priori knowledge of shock locations, since no biasing should occur in smooth regions. Hence, in this paper, we propose two options to automate the stencil choices for the post-processor. The first technique borrows ENO (essentially non-oscillatory) constructions to find the smoothest stencil for post-processing. The second technique utilizes a local edge detection (LED) method developed by Archibald, Gelb, and Yoon [1, 2] to find the shock location, and determines the post-processing stencil based on the proximity of the shock. This study analyzes the behavior of these stencil choosing procedures for both stationary and moving shocks on a series of different uniform meshes and numerically evaluates their use on four scalar problems.

This paper is organized as follows. In Sec. 2 we review the discontinuous Galerkin method and the symmetric and one-sided post-processing technique utilized in this paper. The ENO and LED methods for choosing post-processing stencils are discussed in Sec. 3. Finally, in Sec. 4 we present numerical results demonstrating the benefits of using either of these techniques for choosing the appropriate post-processing stencil.

\section{THE DISCONTINUOUS GALERKIN METHOD AND POST-PROCESSING}

In this section, we give a brief review of the discontinuous Galerkin method, developed by Cockburn and Shu et al. [5, 6, 9, 10, 11], for treating non-linear problems, and the related post-processing technique, introduced by Cockburn, Luskin, Shu and Süli $[4,7,8,12]$. The authors show that the post-processor nearly doubles the order of accuracy for time-dependent linear hyperbolic systems solved over a locally uniform mesh. In addition, numerical evidence strongly indicates that the method also improves the convergence for non-linear problems as well. The applications of the post-processor were extended in [19] to include multi-domains with different mesh sizes as well as variable and discontinuous coefficient equations. The extension of this technique to one-sided post-processing appears in [20]. Detailed discussion of the post-processor, including implementation, can be found in $[7,8,19,20]$. 


\subsection{The Discontinuous Galerkin Method (DGM)}

We begin by considering the discontinuous Galerkin method for the one-dimensional conservation law

$$
u_{t}+f(u)_{x}=0 .
$$

The mesh is defined by $\triangle x_{i}=x_{i+\frac{1}{2}}-x_{i-\frac{1}{2}}$ where $I_{i}=\left(x_{i}-\frac{\Delta x_{i}}{2}, x_{i}+\frac{\Delta x_{i}}{2}\right)$, and $x_{i}$ denotes the cell center, $i=1, \ldots, N$. The approximation space consists of piecewise polynomials of degree less than or equal to $k$, where $k+1$ is the order of accuracy of the approximation, that is, $V_{h}=\{v \mid v \in$ $\mathbb{P}^{k}$ for $x \in I_{i}$. The discontinuous Galerkin method is found by multiplying Eq. (2.1) by a test function $v \in V_{h}$ and integrating by parts to obtain the variational formulation:

Find $u_{h}(x, t) \in V_{h}$ such that

$$
\int_{I_{i}} u_{t} v d x=\int_{I_{i}} f(u) v_{x} d x-f\left(u_{i+1 / 2}\right) v_{i+1 / 2}+f\left(u_{i-1 / 2}\right) v_{i-1 / 2} \quad \forall v \in V_{h} .
$$

The numerical scheme is then given by

$$
\int_{I_{i}}\left(u_{h}\right)_{t} v d x=\int_{I_{i}} f\left(u_{h}\right) v_{x} d x-\hat{f}_{i+1 / 2} v_{i+1 / 2}^{-}+\hat{f}_{i-1 / 2} v_{i-1 / 2}^{+}
$$

for all test functions $v \in V_{h}$. The numerical flux, $\hat{f}_{i+1 / 2}=\hat{f}\left(u_{i+1 / 2}^{-}, u_{i+1 / 2}^{+}\right)$, is chosen to be an upwind monotone flux, i.e. it is a non-decreasing function of the first argument $u^{-}$and a non-increasing function of the second argument $u^{+}$. The test function $v$ is taken from inside the cell. We note that numerical integration of (2.2) by the third-order SSP Runge-Kutta method (see e.g. $[14,15,21])$ guarantees the order of accuracy to agree with the spatial discretization error.

\subsection{Post-processing for DGM}

It was shown in [8] for linear hyperbolic equations that the negativeorder norm error estimate, which provides information about the oscillatory nature of the error, is of higher order than the usual $L_{2}$ norm error estimate for the DGM. The post-processing technique in [20] both reduces oscillations as well as improves accuracy in the $L_{2}$ norm. In fact it is possible to enhance the overall accuracy to be up to the order of the error estimate in the negative-order norm. In addition, the post-processor can be numerically simplified if a locally uniform mesh is assumed, yielding translation invariance and subsequently localizing the post-processor. This assumption will be used in our study, resulting in a post-processor of the form 


$$
u^{*}(x)=\frac{1}{h} \int_{-\infty}^{\infty} K^{2(k+1), k+1}\left(\frac{y-x}{h}\right) u_{h}(y) d y,
$$

where $K^{2(k+1), k+1}$ is a linear combination of B-splines and $u_{h}$ is the numerical solution. The symmetric form of the post-processing kernel can be written as

$$
K^{2(k+1), k+1}(x)=\sum_{\gamma=-k}^{k} c_{\gamma}^{2(k+1), k+1} \psi^{(k+1)}(x-\gamma),
$$

where $\psi^{(k+1)}(x)$ is obtained by convolving the characteristic function over the interval $\left(-\frac{1}{2}, \frac{1}{2}\right)$ with itself $k$ times and $c_{\gamma}^{2(k+1), k+1} \in \mathbb{R}$. The calculation of $c_{\gamma}^{2(k+1), k+1}$ as well as the construction and implementation of (2.3) is described in [3, 19, 20, 22, 23].

We note that since we are using an uniform mesh, the kernel (2.4) has a particularly simple form as in $[7,8,19,20]$. Furthermore, the postprocessor as well as the B-spline coefficients are symmetric. Finally there is an additional advantage in the local behavior of the post-processor, specifically that the kernel needs information only from its nearest neighbors.

The symmetric version of the post-processed solution in (2.3) uses $2 k+1$ B-splines, giving a total support of $2 k^{\prime}+1$ cells where $k^{\prime}=\left\lceil\frac{3 k+1}{2}\right\rceil$. A one-sided version of this post-processor is performed by simply moving the support bias to one-side. For example, a purely left-sided postprocessed solution would be of the form

$$
u^{*}(x)=\frac{1}{h} \int_{-\infty}^{\infty} \sum_{\gamma=-3 k}^{-k} c_{\gamma}^{2(k+1), k+1} \psi^{(k+1)}\left(\frac{y-x}{h}-\gamma\right) u_{h}(y) d y
$$

for $k=1$ or $k=2$. This purely one-sided post-processor has a form similar to the centered one, just with different bounds on the summation and new coefficients $c_{\gamma}^{2 k+1,2 k}$ [20]. Similarly, a partially left-sided post-processor is obtained by changing the bounds of the summation. In each case, the use of $2 k+1 \mathrm{~B}$-splines remains consistent. The right-sided post-processed solution is a mirror image of the left.

\section{POST-PROCESSING STENCIL OPTIONS}

As explained in [20], advanced knowledge of the shock locations is required to determine the post-processing stencil best suited for reconstruction in a shock vicinity. A stencil defines a collection of points $x_{j-l}, \ldots, x_{j+k}$ surrounding the point $x_{j}$ where we wish to determine the 
smoothness of the solution. Because of the considerations outlined in previous sections, we are only considering stencils of equally spaced points. We also assume that the mesh is sufficiently well resolved so that the shocks are well separated and therefore there is a sufficient number of points between shocks.

There are $2 k^{\prime}+1$ candidate stencils for the post-processor in each considered region, such as the five point candidate post-processing stencils for piecewise linear polynomials shown in Fig. 1. An admissible stencil for the post-processing procedure avoids points containing shock values. To this end, here we present two alternative techniques for determining suitable stencils for post-processing the computed numerical solution. The ENO approach chooses the post-processor stencil based on the pattern of the overall smoothness of qualifying stencils. Alternatively, the LED method finds the shock locations directly and then dictates the post-processing stencil to avoid any shocks.

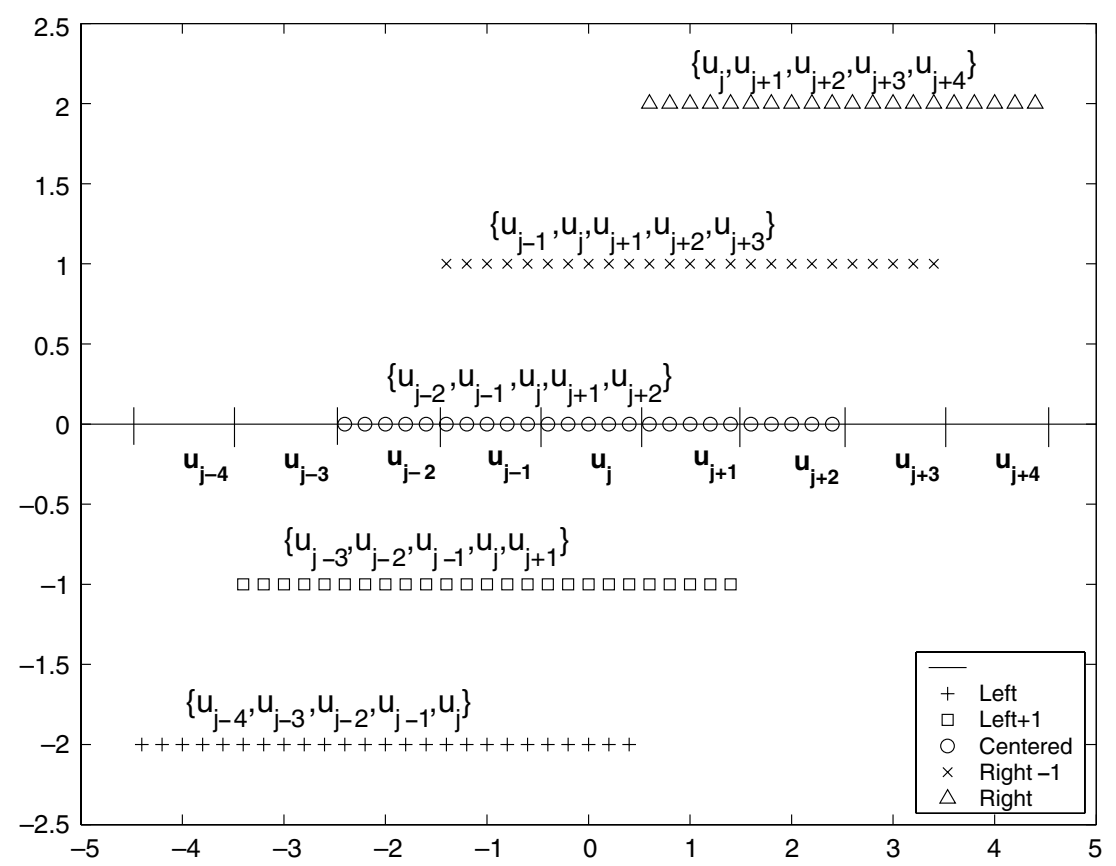

Fig. 1. Five point candidate post-processing stencil choices using a piecewise $\mathbb{P}^{1}$-polynomial approximation. 


\subsection{ENO Type Stencil Choosing}

The essentially non-oscillatory (ENO) finite difference method for hyperbolic equations with discontinuities approximates the values at each $x_{j}$ by interpolating values only in identified smooth regions $[16,17,18,21]$. The ENO stencil choosing technique for the DGM post-processor developed here similarly seeks to evaluate the numerical solution based on the identified smoothest stencil, and then chooses a one-sided, partially one-sided, or a centered stencil to post-process the solution. Unlike the standard ENO algorithm, here we impose a bias toward centered stencils for the DGM solution post-processor since the magnitude of the error is reduced more significantly than if we use a one-sided stencil. (Note that $2 k+1$ order accuracy is still obtained throughout the domain, including regions where one-sided post-processing is implemented.) Below is a description of the ENO type stencil construction process.

Let us first consider the case where $k=1$ in which a third order postprocessor is used. Given the data $u_{j}$ at all of the cell faces, we employ the recursive formula for $r=1,2,3$ :

$$
\begin{aligned}
& D_{i}^{1}=\left|u_{i+1}\left(x_{i-1 / 2}\right)-u_{i}\left(x_{i-1 / 2}\right)\right| \\
& D_{i}^{r+1}=\left|D_{i+1}^{r}-D_{i}^{r}\right|,
\end{aligned}
$$

where $i=j-4, \ldots, j+4-r$. Clearly $D_{j}^{1}$ is the difference between the downwind points across each cell $I_{j}$. Based on this difference, we make the following stencil choice, $S(j)$, for reconstruction at each cell $I_{j}$ :

$$
S(j)=S_{2}(j)=\left\{\begin{array}{cl}
1 & \text { if } N P_{j}^{1}=4 \\
-1 & \text { if } N P_{j}^{1}=5 \\
0 & \text { else }
\end{array}\right.
$$

where $N P_{j}^{r}$ is defined as the value for which $j-4+N P_{j}^{r}$ maximizes the $r$ th difference, i.e.

$$
D_{j-4+N P_{j}^{r}}^{r-1}=\max _{j-4 \leqslant J \leqslant j+4-r} D_{J}^{r-1} .
$$

Table I displays the possible stencil choices when $S(j)=S_{2}(j)$. Although employing the maximum undivided difference yields post-processing stencils without shocks, the stencil might still include cells in which steep gradients of the solution exist, reducing the the overall accuracy of the method. This can be alleviated by implementing a stencil that considers both the first and second undivided difference. Namely, 
Table I. Stencils and stencil numbers for $k=1$

$$
\begin{aligned}
& \begin{array}{rrl}
\hline S(j) & \multicolumn{1}{c}{\text { Stencil }} & \multicolumn{1}{c}{\text { Bias }} \\
\hline & \text { Stencils for } k=1 \\
-1 & \left\{u_{j-4}, u_{j-3}, u_{j-2}, u_{j-1}, u_{j}\right\} & \text { Left } \\
-2 & \left\{u_{j-3}, u_{j-2}, u_{j-1}, u_{j}, u_{j+1}\right\} & \text { Left }+1 \\
0 & \left\{u_{j-2}, u_{j-1}, u_{j}, u_{j+1}, u_{j+2}\right\} & \text { Centered } \\
2 & \left\{u_{j-1}, u_{j}, u_{j+1}, u_{j+2}, u_{j+3}\right\} & \text { Right }-1 \\
1 & \left\{u_{j}, u_{j+1}, u_{j+2}, u_{j+3}, u_{j+4}\right\} & \text { Right } \\
\hline
\end{array} \\
& S(j)=S_{32}(j)= \begin{cases}S_{2}(j) & \text { if } N P_{j}^{2}=2 \text { and } N P_{j}^{1}=3, \\
S_{2}(j) & \text { if } N P_{j}^{2}=3, N P_{j}^{2}=4, N P_{j}^{2}=5 \\
S_{2}(j) & \text { if } N P_{j}^{2}=6 \text { and } N P_{j}^{1}=6, \\
0 & \text { else. }\end{cases}
\end{aligned}
$$

The $S_{32}(j)$ stencil examines the seven options of maximal 3-point stencils and compares them to the maximal two point stencil. To refine this further, we consider the first three differences:

$$
S(j)=S_{432}(j)= \begin{cases}S_{32}(j) & \text { if } N P_{j}^{3}=1 \text { and } 1 \leqslant N P_{j}^{2} \leqslant 2, \\ S_{32}(j) & \text { if } N P_{j}^{3}=2 \text { and } 2 \leqslant N P_{j}^{2} \leqslant 3, \\ S_{32}(j) & \text { if } N P_{j}^{3}=3 \text { and } 3 \leqslant N P_{j}^{2} \leqslant 4, \\ S_{32}(j) & \text { if } N P_{j}^{3}=4 \text { and } 4 \leqslant N P_{j}^{2} \leqslant 5, \\ S_{32}(j) & \text { if } N P_{j}^{3}=5 \text { and } 5 \leqslant N P_{j}^{2} \leqslant 6, \\ S_{32}(j) & \text { if } N P_{j}^{3}=6 \text { and } 6 \leqslant N P_{j}^{2} \leqslant 7 \\ 0 & \text { else, }\end{cases}
$$

or the first four differences:

$$
S(j)=S_{5432}(j)= \begin{cases}S_{432}(j) & \text { if } N P_{j}^{4}=1 \text { and } 1 \leqslant N P_{j}^{3} \leqslant 4, \\ S_{432}(j) & \text { if } N P_{j}^{4}=2 \text { and } 1 \leqslant N P_{j}^{3} \leqslant 5, \\ S_{432}(j) & \text { if } N P_{j}^{4}=3, \\ S_{432}(j) & \text { if } N P_{j}^{4}=4 \text { and } 2 \leqslant N P_{j}^{3} \leqslant 6, \\ S_{432}(j) & \text { if } N P_{j}^{4}=5 \text { and } 3 \leqslant N P_{j}^{3} \leqslant 6, \\ 0 & \text { else. }\end{cases}
$$

Implementing successive levels for $S(j)$ is necessary to provide consistency in determining a suitable post-processing stencil.

The $k=1$ stencil choice essentially finds the discontinuity, and is thus sufficient for suggesting the correct post-processing stencil. Hence, the 
same stencil choosing method (for $k=1$ ) described above can be performed with the information given by the $\mathbb{P}^{k}$-polynomial approximation, for $k \geqslant 2$. Once this skeleton stencil choice-the stencil choice that is based on 5-point stencils-is determined, the 9-point stencil choice easily follows. Specifically, if the 5-point stencil is biased then the 9-point stencil is biased in the same way to avoid the same discontinuous region. The ENO stencil choosing algorithm for $\mathbb{P}^{k}, k=1,2$ is the following:

If $S(j) \neq 0$ then do nothing.

Else if $S(j)=0$ then

If $S(j-1)=1$ then $S(j)=2$.

If $S(j+1)=-1$ then $S(j)=-2$.

If $k \geqslant 2$ then

If $S(j-1)=2$ then $S(j)=3$.

If $S(j-1)=3$ then $S(j)=4$.

If $S(j+2)=-1$ then $S(j)=-3$.

If $S(j+3)=-1$ then $S(j)=-4$.

End If

\section{End If}

Table II displays the stencil options for $k=2$. As is evident from the above algorithm, an advantage of using an ENO stencil choosing technique is that the complexity of the stencil choosing method increases minimally with increasing polynomial degree approximation.

\subsection{A Local Edge Detection Method}

The local edge detection (LED) method used in this study is based on the multivariate edge detection method for scattered data first described

Table II. Stencils and stencil numbers for $k=2$

\begin{tabular}{rcr}
\hline$S(j)$ & Stencil & \multicolumn{1}{c}{ Bias } \\
\hline & Stencils for $k=2$ & \\
-1 & $\left\{u_{j-8}, u_{j-7}, u_{j-6}, u_{j-5}, u_{j-4}, u_{j-3}, u_{j-2}, u_{j-1}, u_{j}\right\}$ & Left \\
-2 & $\left\{u_{j-7}, u_{j-6}, u_{j-5}, u_{j-4}, u_{j-3}, u_{j-2}, u_{j-1}, u_{j}, u_{j+1}\right\}$ & Left +1 \\
-3 & $\left\{u_{j-6}, u_{j-5}, u_{j-4}, u_{j-3}, u_{j-2}, u_{j-1}, u_{j}, u_{j+1}, u_{j+2}\right\}$ & Left +2 \\
-4 & $\left\{u_{j-5}, u_{j-4}, u_{j-3}, u_{j-2}, u_{j-1}, u_{j}, u_{j+1}, u_{j+2}, u_{j+3}\right\}$ & Left +3 \\
0 & $\left\{u_{j-4}, u_{j-3}, u_{j-2}, u_{j-1}, u_{j}, u_{j+1}, u_{j+2}, u_{j+3}, u_{j+4}\right\}$ & Centered \\
4 & $\left\{u_{j-3}, u_{j-2}, u_{j-1}, u_{j}, u_{j+1}, u_{j+2}, u_{j+3}, u_{j+4}, u_{j+5}\right\}$ & Right -3 \\
3 & $\left\{u_{j-2}, u_{j-1}, u_{j}, u_{j+1}, u_{j+2}, u_{j+3}, u_{j+4}, u_{j+5}, u_{j+6}\right\}$ & Right -2 \\
2 & $\left\{u_{j-1}, u_{j}, u_{j+1}, u_{j+2}, u_{j+3}, u_{j+4}, u_{j+5}, u_{j+6}, u_{j+7}\right\}$ & Right -1 \\
1 & $\left\{u_{j}, u_{j+1}, u_{j+2}, u_{j+3}, u_{j+4}, u_{j+5}, u_{j+6}, u_{j+7}, u_{j+8}\right\}$ & Right \\
\hline
\end{tabular}


in [1]. Since here we are considering a uniform mesh, the analytical and computational aspects of the original method are considerably simplified, and essentially the method is reduced to constructing Newton divided difference formulations of various orders. Once the edges are located, the post-processing stencil is simply chosen to avoid the cells that contain jump discontinuities.

Let us denote $J=\{\xi: a \leqslant \xi \leqslant b\}$ as the jump discontinuities of $u(x)$ on $x \in[a, b]$. Each $\xi$ is computed by the LED method to within a grid cell value. Subsequently, the size of the stencil $\left[x_{l}, x_{r}\right]$ used to post-process the solution at the value $x^{*}$ is restricted by

$$
x_{l-1} \leqslant \xi_{v}<x_{l} \leqslant x^{*} \leqslant x_{r} \leqslant \xi_{v+1}<x_{r+1},
$$

for each $\xi_{v} \in J$. In this case, the size of the stencil guaranteeing a smooth region is $r-l+1$, implying that for $u(x) \in C^{k}(x)$ in the region $\left[x_{l}, x_{r}\right]$, we can obtain $2 k+1$ accuracy as long as $2 k^{\prime}+1 \leqslant r-l+1$ (where $k^{\prime}=\left\lceil\frac{3 k+1}{2}\right\rceil$ ), which is in line with the resolution requirements for high order postprocessing discussed in Sec. 2.2. Since the boundary points are defined as jump discontinuities, there is no formal reduction of accuracy as they are approached.

The main difference between the LED stencil construction procedure we describe in this section and the ENO stencil described in Sec. 3.1 is that while the ENO construction chooses candidate stencils based on a perceived smoothness of $u(x)$ in the region of reconstruction, the LED subscribes a particular stencil based directly on the location of the detected jump discontinuities. This procedure has been further extended to identify discontinuities in the derivative in [2], which will be described in Sec. ??. Hence the region of reconstruction can be chosen to avoid discontinuities in the derivative of the function as well.

As in the ENO stencil case, the LED method for one-dimensional data can be formulated using Newton divided differences. We also note that the LED method is completely independent of the order of the postprocessing method since it is based solely on given grid point data. Hence there is no added complexity for higher order post-processing stencils. A brief synopsis is provided here.

\subsubsection{Review of the One-dimensional mth Order LED}

Consider a piecewise smooth function $u(x)$ on $[a, b]$ known at the discrete grid point values $\mathcal{S}=\left\{x_{1}, \ldots, x_{N}\right\}$. We wish to determine whether or not there is a jump discontinuity at any point $x$ in $[a, b]$. Following the development in [1], we construct an edge detector $L_{m} u(x)$ based on a 
stencil $\mathcal{S}_{x}$ containing $m+1$ points around $x$ to be an approximation of the jump function

$$
[u](x):=u\left(x^{+}\right)-u\left(x^{-}\right),
$$

where $u\left(x^{+}\right)$and $u\left(x^{-}\right)$are the right and left hand side limits of $u$ at $x$. Typically in the uniform data case $\mathcal{S}_{x}$ is chosen so that there is an equal distribution of $m+1$ points around $x \in\left[x_{j-1}, x_{j}\right]$, i.e.

$$
\mathcal{S}_{x}=\left\{x_{i-\frac{m}{2}}, \ldots, x_{i+\frac{m}{2}}\right\}
$$

for each $x_{i} \in \mathcal{S}$. If $x$ is near the boundary, the point distribution can be easily modified without affecting the properties of the LED method [1]. To ease notation we re-label the local set $\mathcal{S}_{x}$ for any point $x \in[a, b]$ as

$$
\mathcal{S}_{x}=\left\{x_{1}, \ldots, x_{m+1}\right\} .
$$

Recall that $J=\{\xi: a \leqslant \xi \leqslant b\}$ is the set of jump discontinuities in $[a, b]$ for $u(x)$. The edge detector $L_{m} u(x)$ is characterized by the asymptotic convergence property

$$
L_{m} u(x) \longrightarrow\left\{\begin{array}{ll}
{[u](\xi),} & \text { if } x_{i} \leqslant \xi, x \leqslant x_{i+1} \\
0, & \text { if } I_{x} \cap J=\emptyset
\end{array} \quad \text { for } \xi \in J\right.
$$

where $I_{x}$ is the smallest closed interval such that $\mathcal{S}_{x} \subset I_{x}$.

The general form of the one-dimensional $m$ th order edge detection method is given by

$$
L_{m} u(x)=\frac{1}{q_{m}(x)} \sum_{x_{j} \in \mathcal{S}_{x}} c_{j, \mathcal{S}_{x}}(x) u\left(x_{j}\right), \quad j=1, \ldots, m+1 .
$$

The coefficients $c_{j, \mathcal{S}_{x}}(x)$ are chosen to annihilate polynomials of degree $m$. Hence (3.8) can yield up to $m$ th order accuracy in recovering smooth regions, i.e. where $I_{x} \cap J=\varnothing$, depending on the underlying smoothness of $u(x)$. Specifically, it was shown in [1] that for $\Delta x=\max \left\{\left|x_{i+1}-x_{i}\right|, i=\right.$ $1, \ldots, N\}$, we have

$$
L_{m} u(x)= \begin{cases}{[u](\xi)+\mathcal{O}(\Delta x),} & \text { if } x_{i} \leqslant \xi, x \leqslant x_{i+1} \text { for } \xi \in J, \\ \mathcal{O}\left(\Delta x^{\min (m, k)}\right), & \text { if } u \in C^{k}\left(I_{x}\right) \text { for } k>0 .\end{cases}
$$

We determine $c_{j, \mathcal{S}_{x}}(x)$ by solving the system

$$
\sum_{x_{j} \in \mathcal{S}_{x}} c_{j, \mathcal{S}_{x}}(x) p_{i}\left(x_{j}\right)=p_{i}^{(m)}(x)
$$


Here $p_{i}(x), i=1, \ldots, m$ is a basis of $\mathbb{P}^{m}$, where $\mathbb{P}^{m}$ denotes the space of all polynomials of degree $\leqslant m$. Note that $p_{i}^{(m)}(x)$ is constant for all $m$, and therefore all $c_{j, \mathcal{S}_{x}}(x)$ are also constant. Hence we write $c_{j, \mathcal{S}_{x}}:=c_{j, \mathcal{S}_{x}}(x)$ for each $x_{j} \in \mathcal{S}_{x}$.

The normalization factor $q_{m}(x)$ is set as

$$
q_{m}(x):=\sum_{x_{j} \in \mathcal{S}_{x}^{+}} c_{j, \mathcal{S}_{x}}
$$

where we have defined the sets

$$
\mathcal{S}_{x}^{+}:=\left\{x_{j} \in \mathcal{S}_{x} \mid x_{j} \geqslant x\right\} \text { and } \mathcal{S}_{x}^{-}:=\mathcal{S}_{x} \backslash \mathcal{S}_{x}^{+} .
$$

(Note that $\sum_{x_{j} \in \mathcal{S}_{x}^{+}} c_{j, \mathcal{S}_{x}}=-\sum_{x_{j} \in \mathcal{S}_{x}^{-}} c_{j, \mathcal{S}_{x}}$.)

In the one-dimensional case, (3.8) can be reduced to a Newton divided difference formula [1]. Specifically, by defining

$$
\omega_{j}\left(\mathcal{S}_{x}\right):=\omega_{j}\left(x_{1}, x_{2}, \ldots, x_{k+1}\right)=\prod_{\substack{i=1 \\ i \neq j}}^{m+1}\left(x_{j}-x_{i}\right),
$$

we can directly compute

$$
c_{j, \mathcal{S}_{x}}=\frac{m !}{\omega_{j}\left(\mathcal{S}_{x}\right)}, \quad j=1, \ldots, m+1,
$$

from (3.10) yielding

$$
L_{m} u(x)=\frac{m !}{q_{m}(x)} u\left[\mathcal{S}_{x}\right] .
$$

This is further simplified if the given set of points $\mathcal{S}$ is uniform on $[a, b]$ :

$$
\mathcal{S}:=\left\{x_{i}:=a+i \Delta x \mid i=1, \ldots, N\right\}, \quad \Delta x=\frac{b-a}{N}>0 .
$$

In this case we have

$$
c_{j}=c_{j, \mathcal{S}_{x}}=\frac{m !}{\omega_{j}\left(\mathcal{S}_{x}\right)}=\frac{m !}{h \prod_{i=1, i \neq j}^{m+1}(j-i)}, \quad j=1, \ldots, m+1,
$$

and the corresponding edge detection method

$$
L_{m} u(x)=\frac{1}{q_{m}} \sum_{x_{j} \in \mathcal{S}_{x}} c_{j} u\left(x_{j}\right),
$$

for $q_{m}=\sum_{x_{j} \in \mathcal{S}_{x}^{+}} c_{j}$. It was shown in [1] that $q_{m}(x)$ is never zero. 


\subsubsection{Discontinuities in the Derivative}

Many partial differential equations, including some of the examples in Sec. 4, admit solutions with discontinuities in the derivative of the solution. Since high order reconstruction requires the function to be as smooth as possible, it is desirable to determine all of the jump discontinuities for $u^{(\gamma)}(x)$ where $\gamma=0, \ldots, k$ and $k$ is the order of the reconstruction. In [2] the LED method was extended to compute the jump discontinuities of $u^{(\gamma)}(x)$, namely

$$
\left[u^{(\gamma)}\right](x):=u^{(\gamma)}(x+)-u^{(\gamma)}(x-),
$$

where $u^{(\gamma)}(x+)$ and $u^{(\gamma)}(x-)$ are the right and left side limits of the function $u^{(\gamma)}$ at $x$. The extension of the LED method to detect jump discontinuities in the $\gamma$ derivative is given for one dimension as

$$
L_{m, \gamma, \mathcal{S}_{x}} f(x)=\frac{1}{q_{m, \gamma}\left(x, \mathcal{S}_{x}\right)} \sum_{x_{j} \in \mathcal{S}_{x}} c_{j}\left(x, \mathcal{S}_{x}\right) f\left(x_{j}\right),
$$

with scaling factor

$$
q_{m, \gamma}\left(x, \mathcal{S}_{x}\right):=\sum_{x_{j} \in \mathcal{S}_{x}^{+}} c_{j}\left(x, \mathcal{S}_{x}\right) \frac{\left(x_{j}-x\right)^{\gamma}}{\gamma !}
$$

Here the coefficient are the solution of the linear system

$$
\sum_{x_{j} \in S_{x}} c_{j}\left(x, \mathcal{S}_{x}\right) p_{\ell}\left(x_{j}\right)=p_{\ell}^{(m)}(x), \quad \ell=0, \ldots, m,
$$

subject to the constraints

$$
\sum_{x_{j} \in \mathcal{S}_{x}^{+}} c_{j}\left(x, \mathcal{S}_{x}\right) p_{\ell}\left(x_{j}\right)=\Delta x^{-m+\gamma}\left(x, \mathcal{S}_{x}\right) p_{\ell}^{(\gamma)}(x), \quad \text { for } \ell=0, \ldots, \gamma .
$$

The modification of the scaling factor (3.18) allows the LED method to detect jump discontinuities in the $\gamma$ derivative. The additional constraints (3.20) ensure that the scaling factor is non-zero. Note that the local set $\mathcal{S}_{x}$ must now consist of $(m+\gamma+2)$ points. In [2] it was shown that the linear systems (3.19) and (3.20) admit a unique solution. The resulting approximation (3.17) recovers the jump discontinuities in the $\gamma$ derivative as

$$
\begin{aligned}
& L_{m, \gamma}, \mathcal{S}_{x} u(x) \\
& \quad= \begin{cases}{\left[u^{(\gamma)}\right](\xi)+\mathcal{O}\left(\Delta^{\min (k, m)-\gamma}\right)} & \text { if } x_{j} \leqslant x, \xi \leqslant x_{j+1} \text { for some } \xi \in J_{\gamma}, \\
\mathcal{O}\left(\Delta^{\min (k, m)-\gamma}\right), & \text { if } u \in C^{k}\left(I_{x}\right) \text { for } k>0 .\end{cases}
\end{aligned}
$$


Here

$$
J_{\gamma}=\left\{\xi: u \in C^{\gamma-1}(\xi) \quad \text { and } \quad\left[u^{(\gamma)}\right](\xi) \neq 0\right\}
$$

is the set of $\gamma$ derivative discontinuities. We refer the interested reader to [2] for more details.

The resolution requirement of the modified LED method is that jump discontinuities in the $\gamma$ derivative are separated by a distance greater than $2 \gamma+3$ grid points. In the applications that follow, this resolution requirement is not that prohibitive. Furthermore, as the resolution is increased, the order of the detection method can be increased since the jump discontinuities have more points between them. In the examples that follows, LED is used for $\gamma=0,1$, resulting in the accurate detection of edges and discontinuities in the first derivative. We note that for $\gamma=0$, the constraints (3.20) can be dropped resulting in the original LED formulation (3.8). The parameter $m$ determines the order of the LED method and in these examples takes values between 2 and 5, which is enough to effectively resolve discontinuities while still maintaining the advantages of the LED in low resolution environments. The post-processing reconstruction will only occur in regions that are at least $C^{2}$, yielding at least second order accuracy.

\section{NUMERICAL RESULTS}

We analyzed the ENO type and LED stencil choosing algorithms by testing them on four scalar test cases, each with a DG approximation of polynomial degree $k=1$ and $k=2$. We evaluated the performance of the ENO $S_{32}, S_{432}$ and $S_{5432}$ and the LED stencil choosing methods to demonstrate their respective strength and weaknesses. For each example, we compared the post-processed results from the automated stencil choosing using the ENO $S_{5432}$ and the comparable LED stencil choosing method. We chose to work with the $S_{5432}$ ENO type method as it was the most consistent among the ENO type methods. Three of the sample problems were linear, chosen because the theory only guarantees the order enhancement for the linear case. First the stencil choices were tested on a smooth linear problem in Example 1 to ensure that neither algorithm introduces any errors if used unnecessarily. The second example features two stationary shocks. The third example has two stationary shocks and two moving discontinuities in the derivative. Finally a non-linear problem with a moving discontinuity is presented to demonstrate that the algorithms effectively capture a moving discontinuity.

We present error tables for the numerical solution as well as the first derivative of the solution to demonstrate that the $2 k+1$ order accuracy 
is indeed achieved throughout the domain as long as the discontinuity lies on a cell boundary. The $L_{2}$-errors presented are calculated at the cell centers unless otherwise indicated. Furthermore, we remark that the main advantage of using one-sided post-processing near a discontinuity is in obtaining the $2 k+1$ order accuracy in the first derivative of the solution. As seen in (2.5), the post-processed solution is a polynomial of degree $2 k+1$, which typically would allow us to obtain $2 k+2$ order accuracy. In [7, 8 ] it was shown that $2 k+1$ order accuracy can be obtained for the solution to time-dependent linear hyperbolic equations. This same order accuracy is also achieved in the first derivative for these same equations, as numerically demonstrated in [20].

\section{Example 1: Linear Scalar Convection Equation}

$$
u_{t}+u_{x}=0, \quad 0 \leqslant x \leqslant 2 \pi
$$

with initial condition

$$
u(x, 0)=\sin (x)
$$

is solved to time $T_{f}=12.5$ and the error for the solution is calculated throughout the entire domain. For this problem, all the ENO stencil choosing methods as well as the LED method recognize the smoothness of the solution and thus choose centered post-processing consistently (Fig. 2). The order of accuracy for the solution and the first derivative of the solution is increased from second order to above third order in the $k=1$ case, and from third order to above fifth order in the $k=2$ case (see Table III). This example confirms that the post-processor with automated shock detection does not introduce errors.

\section{Example 2: Linear Discontinuous Coefficient Problem With Two Stationary Shocks}

$$
u_{t}+(a u)_{x}=0
$$

for $-1 \leqslant x \leqslant 1$ where

$$
a(x)= \begin{cases}\frac{1}{2}, & \text { for }-\frac{1}{2} \leqslant x \leqslant \frac{1}{2} \\ 1 & \text { else, }\end{cases}
$$

with initial conditions

$$
u(x, 0)= \begin{cases}-2 \cos (4 \pi x) & \text { for }-\frac{1}{2} \leqslant x \leqslant \frac{1}{2} \\ \cos (2 \pi x) & \text { else. }\end{cases}
$$



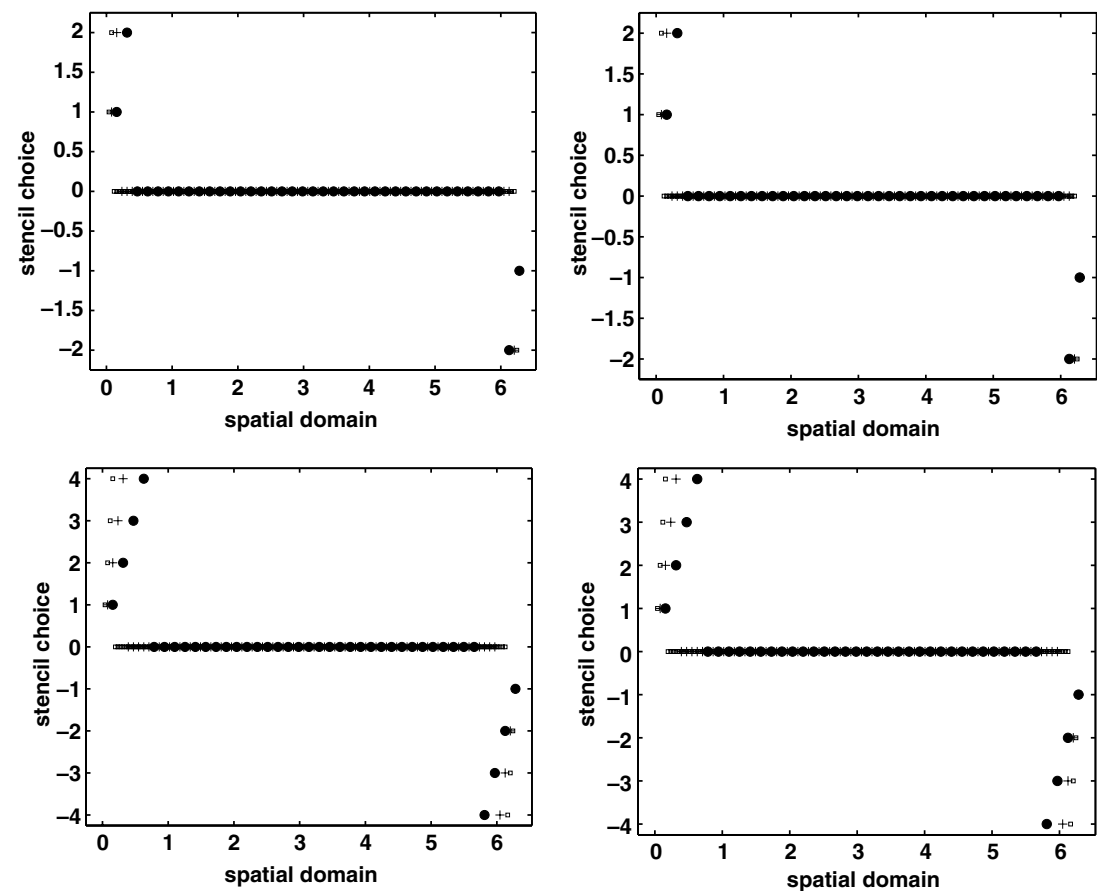

Fig. 2. Example 1 for $k=1$ (top) and $k=2$ (bottom). The stencil choice for the $S_{5432}$ ENO type method is on the left and the LED method is on the right. Both methods correctly choose centered post-processing away from the domain boundary. $N=40=\bullet ; \mathrm{N}=80=+; N=160=\square$.

The exact solution at time $T_{f}=4$ is shown in Fig. 5 (left). This solution features two stationary shocks lying on cell boundaries at $x= \pm \frac{1}{2}$. The numerical solution is computed on a sequence of meshes ranging in resolution from $N=40$ to 160 . The error in the solution and in the first derivative of the solution, before and after post-processing, is calculated at time $T_{f}=4$ over the entire domain and is given in Table IV. For this example, the ENO type methods all bias unnecessarily for the low resolution meshes $N=40$ and 60 . However, the $S_{5432}$ falsely biases less often, and thus gives more reliable stencil choices than the $S_{432}$ and $S_{32}$. The ENO $S_{5432}$ stencil choices for $N=40,80,160$ are plotted in Figs. 3 and 4. For $N=40$ and 60 the errors from the ENO stencil choice is larger than the error from the LED stencil (Table IV), implying that the LED method handles low resolution environments better than the ENO type methods. Once the the grid is sufficiently refined, all four methods identically yield the correct stencil choices.

Since the discontinuities are located at the cell boundaries, the discontinuous Galerkin method can produce $2 k+1$ order accuracy in the post-processed numerical solution and its first derivative throughout the 
Table III. The $L_{2}$ error and order of accuracy for the solution of Example 1 calculated with $k=1$ and $k=2$

\begin{tabular}{|c|c|c|c|c|c|c|}
\hline \multirow[b]{2}{*}{$N$} & \multicolumn{2}{|c|}{ Before } & \multicolumn{2}{|c|}{$S_{5432}$} & \multicolumn{2}{|c|}{ LED } \\
\hline & $L_{2}$ error & Order & $L_{2}$ error & Order & $L_{2}$ error & Order \\
\hline & \multicolumn{6}{|c|}{ Errors in solution } \\
\hline \multicolumn{7}{|l|}{$k=1$} \\
\hline 40 & 1.1994E-03 & - & $8.4052 \mathrm{E}-04$ & - & $8.4052 \mathrm{E}-04$ & - \\
\hline 60 & $4.6352 \mathrm{E}-04$ & 2.34 & $2.2290 \mathrm{E}-04$ & 3.27 & $2.2290 \mathrm{E}-04$ & 3.27 \\
\hline 80 & $2.4106 \mathrm{E}-04$ & 2.27 & $8.7144 \mathrm{E}-05$ & 3.26 & $8.7145 \mathrm{E}-05$ & 3.26 \\
\hline 100 & $1.4670 \mathrm{E}-04$ & 2.23 & $4.2253 \mathrm{E}-05$ & 3.24 & $4.2253 \mathrm{E}-05$ & 3.24 \\
\hline 120 & $9.8368 \mathrm{E}-05$ & 2.19 & $2.3474 \mathrm{E}-05$ & 3.22 & $2.3474 \mathrm{E}-05$ & 3.22 \\
\hline 140 & $7.0427 \mathrm{E}-05$ & 2.17 & $1.4321 \mathrm{E}-05$ & 3.21 & $1.4321 \mathrm{E}-05$ & 3.21 \\
\hline 160 & $5.2861 \mathrm{E}-05$ & 2.15 & $9.3540 \mathrm{E}-06$ & 3.19 & $9.3541 \mathrm{E}-06$ & 3.19 \\
\hline \multicolumn{7}{|l|}{$k=2$} \\
\hline 40 & $1.1404 \mathrm{E}-05$ & - & $8.4145 \mathrm{E}-05$ & - & $1.0557 \mathrm{E}-05$ & - \\
\hline 60 & $3.3814 \mathrm{E}-06$ & 3.00 & $1.0195 \mathrm{E}-05$ & 5.21 & $1.2234 \mathrm{E}-06$ & 5.32 \\
\hline 80 & $1.4269 \mathrm{E}-06$ & 3.00 & $2.1801 \mathrm{E}-06$ & 5.36 & $2.5790 \mathrm{E}-07$ & 5.41 \\
\hline 100 & $7.3066 \mathrm{E}-07$ & 3.00 & $6.5063 \mathrm{E}-07$ & 5.42 & $7.6474 \mathrm{E}-08$ & 5.45 \\
\hline 120 & $4.2287 \mathrm{E}-07$ & 3.00 & $2.4103 \mathrm{E}-07$ & 5.45 & $2.8234 \mathrm{E}-08$ & 5.47 \\
\hline 140 & $2.6631 \mathrm{E}-07$ & 3.00 & $1.0385 \mathrm{E}-07$ & 5.46 & $1.2141 \mathrm{E}-08$ & 5.48 \\
\hline 160 & $1.7841 \mathrm{E}-07$ & 3.00 & $5.0013 \mathrm{E}-08$ & 5.47 & $5.8398 \mathrm{E}-09$ & 5.48 \\
\hline
\end{tabular}

Results are shown before and after post-processing determined by stencil choices $S_{5432}$ and LED.

domain, as long as the stencil choice is correct (Table IV). As in [20], the errors for the numerical solution are better than the expected $2 k+1$ order accuracy.

\section{Example 3: Linear Discontinuous Coefficient Problem With Two Stationary Shocks and Two Moving Shocks}

$$
u_{t}+(a u)_{x}=0
$$

for $-2 \leqslant x \leqslant 2$ where

$$
a(x)= \begin{cases}\frac{1}{2} & \text { for }-1 \leqslant x \leqslant 1 \\ 1 & \text { else }\end{cases}
$$

with initial condition,

$$
u(x, 0)= \begin{cases}\frac{2}{3} \sin (\pi x) & \text { for }-1 \leqslant x \leqslant 1, \\ \cos \left(\frac{1}{2} \pi x\right) & \text { else. }\end{cases}
$$


Table IV. The $L_{2}$ errors and order of accuracy for the solution (top) and first derivative of the solution (bottom) for Example 2, calculated with $k=1$ and $k=2$.

\begin{tabular}{|c|c|c|c|c|c|c|}
\hline \multirow[b]{2}{*}{$N$} & \multicolumn{2}{|c|}{ Before } & \multicolumn{2}{|c|}{$S_{5432}$} & \multicolumn{2}{|c|}{ LED } \\
\hline & $L_{2}$ error & Order & $L_{2}$ error & Order & $L_{2}$ error & Order \\
\hline & \multicolumn{6}{|c|}{ Errors in solution } \\
\hline \multicolumn{7}{|l|}{$k=1$} \\
\hline 40 & $8.0432 \mathrm{E}-02$ & - & $2.2491 \mathrm{E}-01$ & - & $8.4815 \mathrm{E}-02$ & - \\
\hline 60 & $2.7136 \mathrm{E}-02$ & 2.68 & $2.3724 \mathrm{E}-02$ & 5.55 & $2.2551 \mathrm{E}-02$ & 3.27 \\
\hline 80 & $1.2584 \mathrm{E}-02$ & 2.67 & $8.9582 \mathrm{E}-03$ & 3.39 & $8.9582 \mathrm{E}-03$ & 3.21 \\
\hline 100 & $6.9905 \mathrm{E}-03$ & 2.63 & 4.4844E-03 & 3.10 & 4.4844E-03 & 3.10 \\
\hline 120 & $4.3553 \mathrm{E}-03$ & 2.60 & $2.5753 \mathrm{E}-03$ & 3.04 & $2.5753 \mathrm{E}-03$ & 3.04 \\
\hline 140 & $2.9360 \mathrm{E}-03$ & 2.56 & $1.6183 \mathrm{E}-03$ & 3.01 & $1.6183 \mathrm{E}-03$ & 3.01 \\
\hline 160 & $2.0959 \mathrm{E}-03$ & 2.52 & $1.0841 \mathrm{E}-03$ & 3.00 & $1.0841 \mathrm{E}-03$ & 3.00 \\
\hline \multicolumn{7}{|l|}{$k=2$} \\
\hline 40 & $1.0663 \mathrm{E}-03$ & - & $7.4252 \mathrm{E}-02$ & - & $2.0510 \mathrm{E}-02$ & - \\
\hline 60 & $3.0828 \mathrm{E}-04$ & 3.06 & $1.8714 \mathrm{E}-02$ & 3.40 & $2.4536 \mathrm{E}-03$ & 5.24 \\
\hline 80 & $1.2955 \mathrm{E}-04$ & 3.01 & $4.2724 \mathrm{E}-03$ & 5.13 & $4.4396 \mathrm{E}-04$ & 5.94 \\
\hline 100 & $6.6271 \mathrm{E}-05$ & 3.00 & $1.1202 \mathrm{E}-03$ & 6.00 & $1.1186 \mathrm{E}-04$ & 6.18 \\
\hline 120 & $3.8343 \mathrm{E}-05$ & 3.00 & $3.6331 \mathrm{E}-04$ & 6.18 & $3.5549 \mathrm{E}-05$ & 6.29 \\
\hline 140 & $2.4145 \mathrm{E}-05$ & 3.00 & $1.3816 \mathrm{E}-04$ & 6.27 & $1.3361 \mathrm{E}-05$ & 6.35 \\
\hline 160 & $1.6176 \mathrm{E}-05$ & 3.00 & $5.9332 \mathrm{E}-05$ & 6.33 & $5.6958 \mathrm{E}-06$ & 6.39 \\
\hline & \multicolumn{6}{|c|}{ Errors in first derivative of the solution } \\
\hline \multicolumn{7}{|c|}{ 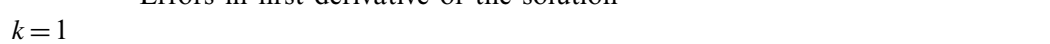 } \\
\hline 40 & $1.5086 \mathrm{E}+00$ & - & $3.0423 \mathrm{E}+00$ & - & $8.4586 \mathrm{E}-01$ & - \\
\hline 60 & $9.0952 \mathrm{E}-01$ & 1.25 & $3.2012 \mathrm{E}-01$ & 5.55 & $3.0056 \mathrm{E}-01$ & 2.55 \\
\hline 80 & $6.6871 \mathrm{E}-01$ & 1.07 & $1.3100 \mathrm{E}-01$ & 3.11 & $1.3100 \mathrm{E}-01$ & 2.89 \\
\hline 100 & $5.3212 \mathrm{E}-01$ & 1.02 & $6.6682 \mathrm{E}-02$ & 3.03 & $6.6682 \mathrm{E}-02$ & 3.03 \\
\hline 120 & 4.4264E-01 & 1.01 & $3.8018 \mathrm{E}-02$ & 3.08 & $3.8018 \mathrm{E}-02$ & 3.08 \\
\hline 140 & $3.7914 \mathrm{E}-01$ & 1.00 & $2.3561 \mathrm{E}-02$ & 3.10 & $2.3561 \mathrm{E}-02$ & 3.10 \\
\hline 160 & $3.3165 \mathrm{E}-01$ & 1.00 & $1.5552 \mathrm{E}-02$ & 3.11 & $1.5552 \mathrm{E}-02$ & 3.11 \\
\hline \multicolumn{7}{|l|}{$k=2$} \\
\hline 40 & $1.2741 \mathrm{E}-01$ & - & $3.5888 \mathrm{E}+00$ & - & $1.0608 \mathrm{E}-01$ & - \\
\hline 60 & $5.5669 \mathrm{E}-02$ & 2.04 & $2.3168 \mathrm{E}+00$ & 1.08 & $8.3199 \mathrm{E}-03$ & 6.28 \\
\hline 80 & $3.1175 \mathrm{E}-02$ & 2.02 & $1.9245 \mathrm{E}-02$ & 16.65 & $3.7412 \mathrm{E}-03$ & 2.78 \\
\hline 100 & $1.9920 \mathrm{E}-02$ & 2.01 & $9.4099 \mathrm{E}-03$ & 3.21 & $1.4121 \mathrm{E}-03$ & 4.37 \\
\hline 120 & $1.3823 \mathrm{E}-02$ & 2.00 & $4.2776 \mathrm{E}-03$ & 4.32 & $5.8566 \mathrm{E}-04$ & 4.83 \\
\hline 140 & $1.0152 \mathrm{E}-02$ & 2.00 & $2.0576 \mathrm{E}-03$ & 4.75 & $2.6923 \mathrm{E}-04$ & 5.04 \\
\hline 160 & $7.7706 \mathrm{E}-03$ & 2.00 & $1.0601 \mathrm{E}-03$ & 4.97 & $1.3513 \mathrm{E}-04$ & 5.16 \\
\hline
\end{tabular}

Results are shown for before and after post-processing with stencil choices determined by the $S_{5432}$ and the LED method. 

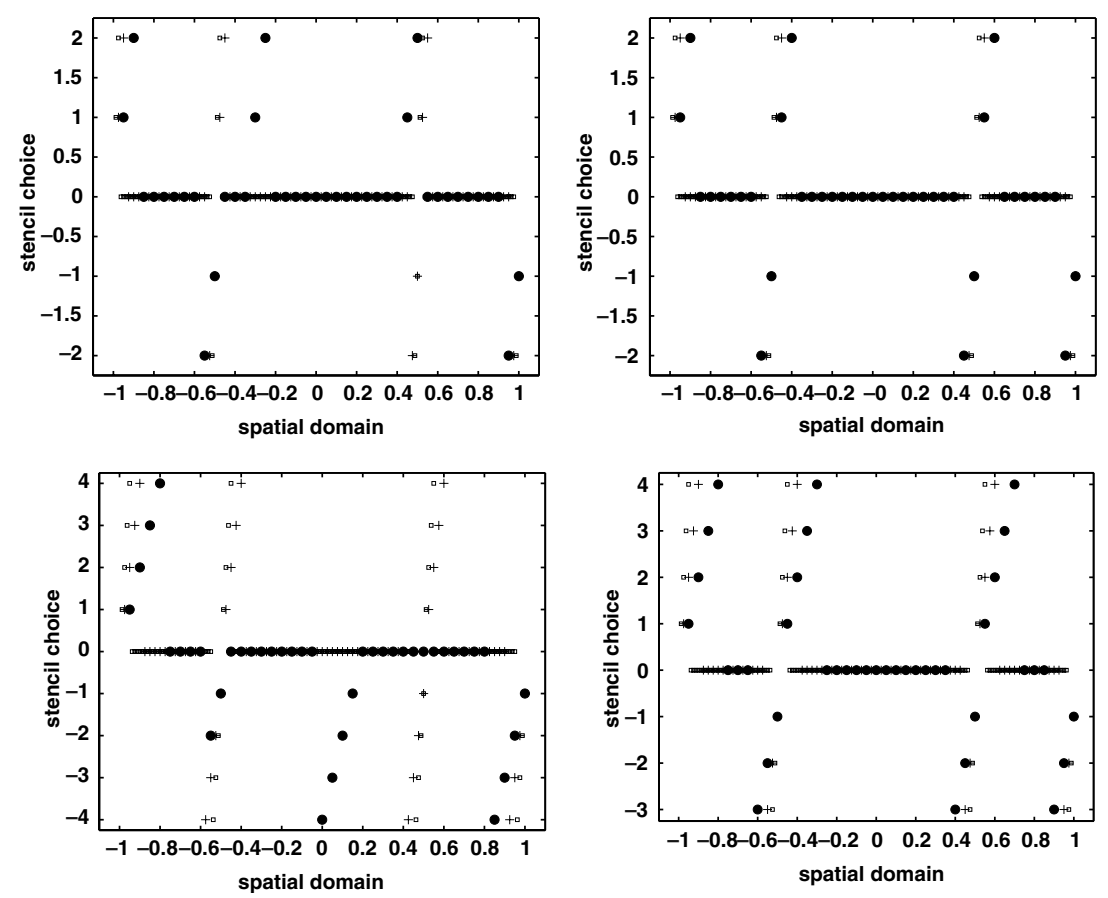

Fig. 3. Example 2 with $k=1$ (top) and $k=2$ (bottom). The LED stencil choosing (right) is able to handle low resolution better than the $S_{5432}$ ENO type method (left). Both the $S_{5432}$ and LED methods are able to detect the stationary shocks at $x= \pm \frac{1}{2} \cdot N=40=\bullet ; \quad \mathrm{N}=80=+$; $N=160=\square$.

The solution at time $T_{f}=1.0$, shown in Fig. 5 (right) features two stationary and two moving shocks where the stationary shocks at $x= \pm 1$ are located at cell boundaries and one moving shock is located near the right boundary and the other moving shock is located near the stationary shock at $x=-1$. The solution is computed to time $T_{f}=1.0$, which is before the shocks cross. The error in the numerical solution and in the first derivative of the solution is calculated to include the stationary shocks at $x= \pm 1$, but to exclude a radius of 0.1 around the moving shocks as in $[19,20]$.

For $k=1$, all of the ENO type stencil choices $\left(S_{32}, S_{432}\right.$ and $\left.S_{5432}\right)$, as well as the LED method, detect the stationary shocks reliably even for low mesh resolution. However, the moving shock in the middle of the domain, which has a discontinuity only in the derivative, is not detected by the ENO stencil choices, but is detected by the LED method. For low resolution the two shocks (the stationary and moving shocks) are too close together for a stencil between them. Thus, we are only interested in the case of $N \geqslant 60$. In this case, the LED method is clearly superior to the 

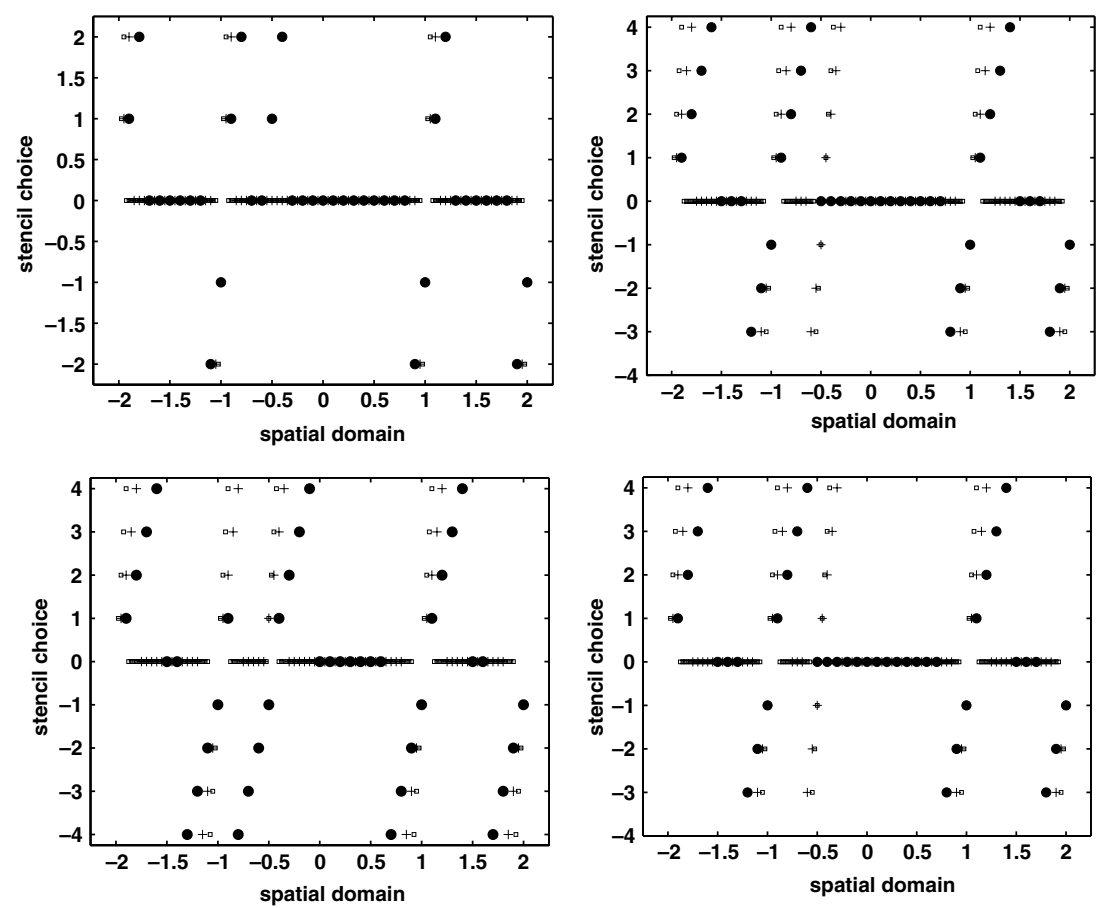

Fig. 4. Example 3 with $k=1$ (top) and $k=2$ (bottom). Both the ENO- $S_{5432}$ (left) and LED (right) methods detect the stationary and moving shocks for $k=2$, but the ENO method has difficulty detecting the moving shocks for $k=1 . N=40=\bullet ; N=80=+; N=160=\square$.
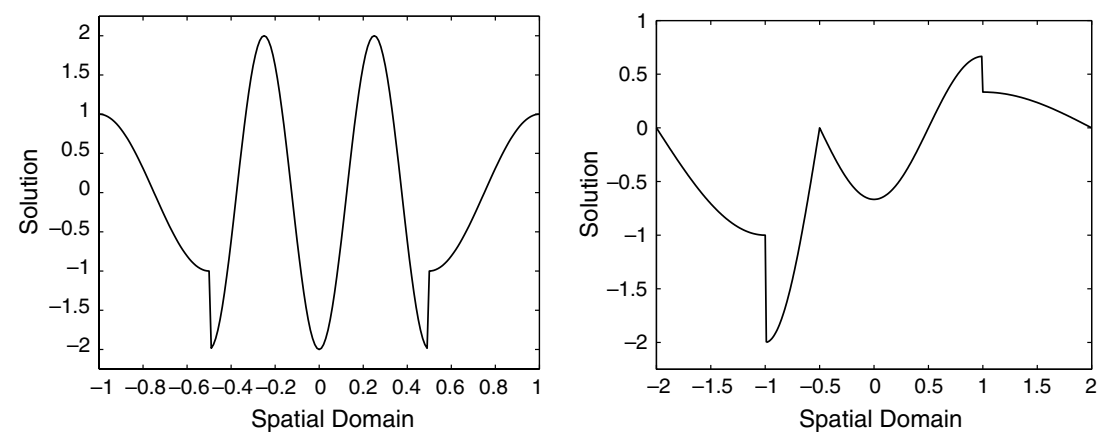

Fig. 5. Numerical solutions for Example 2 (left) and Example 3 (right).

ENO type stencil choices. However, as we are excluding a region of 0.1 around the shock, this advantage is not evident in the error tables. For $k=2$ the ENO type stencil choices $\left(S_{32}, S_{432}\right.$, and $\left.S_{5432}\right)$ and the LED 
method are all equivalent and all capture the correct shock location for all resolutions.

These stencil choices allow the post-processor to both decrease the magnitude of error and increase the order of accuracy. Table $\mathrm{V}$ shows that the order of accuracy of the post-processed solution was raised from three to above six. Again we note that the post-processor gives better than expected errors for the solution, similar to those shown in [20]. In this case, the ENO stencil choosing method may have trouble in the presence of a discontinuity in the derivative, as this method is not designed to determine the locations of discontinuities in the derivative. Remarkably, this method does detect the discontinuity in the solution for the $k=2$ case. The extension of the ENO type stencil choosing methods to cases of discontinuities in the derivative will be addressed in future work.

\section{Example 4: Burgers' Equation}

$$
u_{t}+\left(\frac{1}{2} u^{2}\right)_{x}=0, \quad 0 \leqslant x \leqslant 2 \pi,
$$

with initial conditions

$$
u(x, 0)=\frac{1}{2}+\sin (x)
$$

is solved to time $T_{f}=2.0$, after a shock has formed as in [8]. This example features a moving discontinuity which all of the ENO stencil choices as well as the LED method capture for all choices of $N$ (Fig. 6).

\section{CONCLUSIONS}

We have presented two effective methods for finding the appropriate post-processing stencil. The ENO type stencil choosing is designed to pick centered post-processing in smooth regions and one-sided or partially one-sided post-processing in the neighborhood of a discontinuity while the LED method directly locates the shocks in the numerical solution to determine the correct post-processing stencil.

The ENO type stencil choosing is based on the smoothness of the candidate stencils and is determined by examining the first four undivided differences over the candidate stencils. Implementation of the successive levels is necessary to avoid one-sided post-processing in regions where steep gradients in the solution exist. This type of stencil choosing method requires minimal complexity as the polynomial degree used in the approximation is increased. 
Table V. The $L_{2}$ errors and order of accuracy for the solution and first derivatives of the solution for Example 3 which features two moving and two stationary shocks, calculated with $k=1$ and $k=2$.

\begin{tabular}{|c|c|c|c|c|c|c|}
\hline \multirow[b]{2}{*}{$N$} & \multicolumn{2}{|c|}{ Before } & \multicolumn{2}{|c|}{$S_{5432}$} & \multicolumn{2}{|c|}{ LED } \\
\hline & $L_{2}$ error & Order & $L_{2}$ error & Order & $L_{2}$ error & Order \\
\hline & \multicolumn{6}{|c|}{ Errors in solution } \\
\hline \multicolumn{7}{|c|}{ 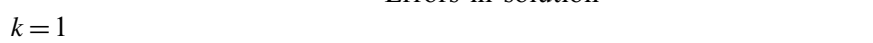 } \\
\hline 40 & $1.0969 \mathrm{E}-03$ & - & $2.0388 \mathrm{E}-03$ & - & $1.0107 \mathrm{E}-02$ & - \\
\hline 60 & 4.5949E-04 & 2.15 & $3.4209 \mathrm{E}-04$ & 4.40 & $3.4209 \mathrm{E}-04$ & 8.35 \\
\hline 80 & $2.5043 \mathrm{E}-04$ & 2.11 & $9.4821 \mathrm{E}-05$ & 4.46 & $9.4821 \mathrm{E}-05$ & 4.46 \\
\hline 100 & $1.5715 \mathrm{E}-04$ & 2.09 & $3.5083 \mathrm{E}-05$ & 4.46 & $3.5083 \mathrm{E}-05$ & 4.46 \\
\hline 120 & $1.0768 \mathrm{E}-04$ & 2.07 & $1.5693 \mathrm{E}-05$ & 4.41 & $1.5693 \mathrm{E}-05$ & 4.41 \\
\hline 140 & 7.8344E-05 & 2.06 & $8.0425 \mathrm{E}-06$ & 4.34 & $8.0425 \mathrm{E}-06$ & 4.34 \\
\hline 160 & 5.9541E-05 & 2.06 & 4.5692E-06 & 4.23 & $4.5692 \mathrm{E}-06$ & 4.23 \\
\hline \multicolumn{7}{|l|}{$k=2$} \\
\hline 40 & $3.1528 \mathrm{E}-05$ & - & $1.8373 \mathrm{E}-03$ & - & $1.9032 \mathrm{E}-04$ & - \\
\hline 60 & $9.4838 \mathrm{E}-06$ & 2.96 & $1.5620 \mathrm{E}-04$ & 6.07 & $1.5224 \mathrm{E}-05$ & 6.23 \\
\hline 80 & $4.0275 \mathrm{E}-06$ & 2.98 & $2.5501 \mathrm{E}-05$ & 6.30 & $2.4366 \mathrm{E}-06$ & 6.37 \\
\hline 100 & $2.0698 \mathrm{E}-06$ & 2.98 & $6.1383 \mathrm{E}-06$ & 6.38 & $5.8134 \mathrm{E}-07$ & 6.42 \\
\hline 120 & $1.2006 \mathrm{E}-06$ & 2.99 & $1.9034 \mathrm{E}-06$ & 6.42 & $1.7942 \mathrm{E}-07$ & 6.45 \\
\hline 140 & 7.5734E-07 & 2.99 & $7.0484 \mathrm{E}-07$ & 6.44 & $6.6257 \mathrm{E}-08$ & 6.46 \\
\hline 160 & 5.0797E-07 & 2.99 & $2.9754 \mathrm{E}-07$ & 6.46 & $2.7922 \mathrm{E}-08$ & 6.47 \\
\hline & \multicolumn{6}{|c|}{ Errors in first derivative } \\
\hline \multicolumn{7}{|c|}{ ש } \\
\hline 40 & 3.8324E-02 & - & 8.7819E-03 & - & $3.0425 \mathrm{E}+01$ & - \\
\hline 60 & $2.5115 \mathrm{E}-02$ & 1.04 & $2.4476 \mathrm{E}-03$ & 3.15 & $2.4476 \mathrm{E}-03$ & 11.89 \\
\hline 80 & $1.8657 \mathrm{E}-02$ & 1.03 & $9.3777 \mathrm{E}-04$ & 3.33 & $9.3777 \mathrm{E}-04$ & 3.33 \\
\hline 100 & $1.4836 \mathrm{E}-02$ & 1.03 & $4.3900 \mathrm{E}-04$ & 3.40 & $4.3900 \mathrm{E}-04$ & 3.40 \\
\hline 120 & $1.2312 \mathrm{E}-02$ & 1.02 & $2.3478 \mathrm{E}-04$ & 3.43 & $2.3478 \mathrm{E}-04$ & 3.43 \\
\hline 140 & $1.0521 \mathrm{E}-02$ & 1.02 & $1.3794 \mathrm{E}-04$ & 3.45 & $1.3794 \mathrm{E}-04$ & 3.45 \\
\hline 160 & $9.1844 \mathrm{E}-03$ & 1.02 & $8.6904 \mathrm{E}-05$ & 3.46 & $8.6904 \mathrm{E}-05$ & 3.46 \\
\hline \multicolumn{7}{|l|}{$k=2$} \\
\hline 40 & $1.9866 \mathrm{E}-03$ & - & $2.0750 \mathrm{E}-03$ & - & $3.9894 \mathrm{E}-04$ & - \\
\hline 60 & $8.7943 \mathrm{E}-04$ & 2.01 & $4.6036 \mathrm{E}-04$ & 3.71 & $6.2260 \mathrm{E}-05$ & 4.58 \\
\hline 80 & 4.9381E-04 & 2.01 & $1.1390 \mathrm{E}-04$ & 4.86 & $1.4300 \mathrm{E}-05$ & 5.11 \\
\hline 100 & $3.1572 \mathrm{E}-04$ & 2.00 & $3.6119 \mathrm{E}-05$ & 5.15 & $4.4020 \mathrm{E}-06$ & 5.28 \\
\hline 120 & $2.1911 \mathrm{E}-04$ & 2.00 & $1.3808 \mathrm{E}-05$ & 5.27 & $1.6575 \mathrm{E}-06$ & 5.36 \\
\hline 140 & $1.6091 \mathrm{E}-04$ & 2.00 & $6.0605 \mathrm{E}-06$ & 5.34 & $7.2110 \mathrm{E}-07$ & 5.40 \\
\hline 160 & $1.2315 \mathrm{E}-04$ & 2.00 & $2.9534 \mathrm{E}-06$ & 5.38 & $3.4945 \mathrm{E}-07$ & 5.43 \\
\hline
\end{tabular}

The errors are calculated outside a radius of 0.1 of the moving shocks. Results are shown before post-processing and after post-processing determined by stencil choices ENO-S5432 and LED methods. 

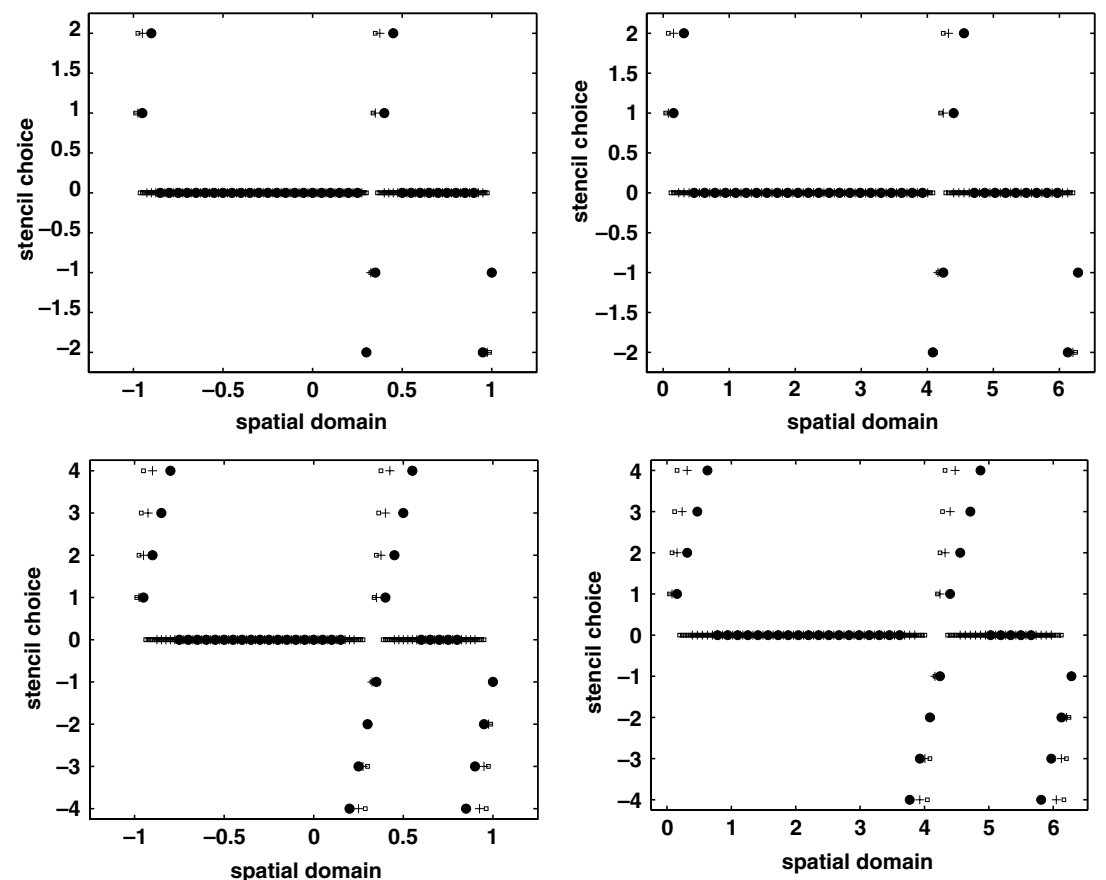

Fig. 6. Example 4 for $k=1$ (top) and $k=2$ (bottom). Neither the ENO- $S_{5432}$ (left) nor LED (right) method has difficulty detecting the moving shock. $N=40=\bullet ; \quad N=80=+$; $N=160=\square$.

The LED method is particularly effective in identifying the shock locations in the case of low resolution, as well as for non-uniform spacing. The algorithm is also effective for determing jump discontinuities and guarantees that post-processing will only occur in smooth regions (at least $C^{2}$ ). The method is completely independent of the post-processing approximation order.

We have demonstrated through numerical examples that both these methods are viable and efficient techniques for automated choice of the post-processing stencil by enabling the designed $(2 k+1)$ th order accuracy of the post-processor throughout the entire domain. No other predetermination of discontinuity locations is necessary.

In the case where the discontinuity is in the derivative, the ENO type stencil choosing method is unreliable, while the LED method works very well. However, we suspect that the ENO type algorithms can be readily adapted for this purpose and will be the subject of future investigations. Future work will include extending these methods to systems, multiple space dimensions, and non-uniform mesh size. 


\section{ACKNOWLEDGEMENTS}

The second author would like to acknowledge the support of NSE grants No. EAR 0222327, DMS 0107428, and CNS 0324957. The fourth author would like to acknowledge the Householder Fellowship in Scientific Computing sponsored by the Department of Energy Applied Mathematical Sciences program. Program of Oak Ridge National Laboratory (ORNL), managed by UT-Battelle, LLC for the U.S. Department of Energy under Contract No. DE-AC05-00Or22725.

\section{REFERENCES}

1. Archibald, R., Gelb, A., and Yoon, J. (2005). Polynomial fitting for edge detection in irregularly sampled signals and images. SIAM J. Numer. Anal. 43, 259-279.

2. Archibald, R., Gelb, A., and Yoon, J. (2006). Determining the locations of the discontinuities in the derivatives of functions. Appl. Numer. Math. (submitted).

3. Bramble, J. H., and Schatz, A. H. (1977). Higher order local accuracy by averaging in the finite element method. Math. Comput. 31, 94-111.

4. Cockburn, B. (1999). Discontinuous Galerkin methods for convection-dominated problems. In Barth, T. J., and Deconinck, H. (ed.), High-Order Methods for Computational Physics, Lecture Notes in Computational Science and Engineering, Vol. 9, Springer, pp. 69-224.

5. Cockburn, B., Hou, S., and Shu, C.-W. (1990). The Runge-Kutta local projection discontinuous Galerkin finite element method for conservation laws IV: The multidimensional case. Math. Comput. 54, 545-581.

6. Cockburn, B., Lin, S.-Y., and Shu, C.-W. (1989). TVB Runge-Kutta local projection discontinuous Galerkin finite element method for conservation laws III: One dimensional systems. J. Comput. Phys. 84, 90-113.

7. Cockburn, B., Luskin, M., Shu, C.-W., and Süli, E. (1999). Post-processing of Galerkin methods for hyperbolic problems. In Karniadakis, G., Cockburn, B., and Shu, C.-W. (eds.), Proceedings of the International Symposium on Discontinuous Galerkin Methods, Lecture Notes in Computational Science and Engineering, Vol. 11, Springer, pp. 291-300.

8. Cockburn, B., Luskin, M., Shu, C.-W., and Süli, E. (2003). Enhanced accuracy by postprocessing for finite element methods for hyperbolic equations. Math. Comput. 72, 577-606.

9. Cockburn, B., and Shu, C.-W. (1989). TVB Runge-Kutta local projection discontinuous Galerkin finite element method for conservation laws II: General framework. Math. Comput. 52, 411-435.

10. Cockburn, B., and Shu, C.-W. (1991). The Runge-Kutta local projection $\mathbb{P}^{1}$-discontinuous-Galerkin finite element method for scalar conservation laws. Math. Model. Numer. Anal. ( $\left.M^{2} A N\right), 25,337-361$.

11. Cockburn, B., and Shu, C.-W. (1998). The Runge-Kutta discontinuous Galerkin method for conservation laws V: Multidimensional systems. J. Comput. Phys. 141, 199-224.

12. Cockburn, B., and Shu, C.-W. (2001). Runge-Kutta Discontinuous Galerkin methods for convection-dominated problems. J. Scientific Comput. 16, 173-261.

13. Gelb, A., and Tadmor, E. (2000). Detection of edges in spectral data II. Nonlinear enhancement. SIAM J. Numer. Anal. 38, 1389-1408.

14. Gottlieb, S., and Shu, C.-W. (1998). Total variation diminishing Runge-Kutta schemes. Math. Comput. 67, 73-85. 
15. Gottlieb, S., Shu, C.-W., and Tadmor, E. (2001). Strong stability preserving high-order time discretization methods, SIAM Rev. 43, 89-112.

16. Harten, A., Engquist, B., Osher, S., and Chakravarthy, S. (1987). Uniformly high order essentially non-oscillatory schemes I. SIAM J. Numer. Anal. 24, 279-309.

17. Harten, A., Engquist, B., Osher, S., and Chakravarthy, S. (1987). Uniformly high order essentially non-oscillatory schemes. J. Comput. Phys. 71, 231-303.

18. Liu, X.-D., Osher, S., and Chan, T. (1994). Weighted essentially non-oscillatory schemes. J. Comput. Phys. 115, 200-212.

19. Ryan, J. K., Shu, C.-W., and Atkins, H. L. Extension of a post-processing technique for the discontinuous Galerkin method for hyperbolic equations with application to an aeroacoustic problem. SIAM J. Scientific Comp. 26, 821-843.

20. Ryan, J. K., and Shu, C.-W. (2003). On a one-sided post-processing technique for the discontinuous Galerkin method. Methods Appl. Anal. 10(2), 295-307.

21. Shu, C.-W., and Osher, S. (1988). Efficient implementation of essentially non-oscillatory shock-capturing schemes. J. Comput. Phys. 77, 439-471.

22. Thomée, V. (1977). High order local approximations to derivatives in the finite element method. Math. Comput. 31, 652-660.

23. Wahlbin, L. B. (1995). Superconvergence in Galerkin Finite Element Methods, Lecture Notes in Mathematics, Vol. 1605, Springer Verlag. 